\title{
Bi-directional Long Short-Term Memory Model with Semantic Positional Attention for the Question Answering System
}

\author{
MINGWEN BI, College of Information and Electrical Engineering, China Agricultural University, Key \\ Laboratory of Agricultural Informatization Standardization, Ministry of Agriculture and Rural Affairs, \\ Beijing, China \\ QINGCHUAN ZHANG, MIN ZUO, ZELONG XU, and QINGYU JIN, National Engineering \\ Laboratory for Agri-product Quality Traceability, Beijing Technology and Business University, Beijing, China
}

\begin{abstract}
The intelligent question answering system aims to provide quick and concise feedback on the questions of users. Although the performance of phrase-level and numerous attention models have been improved, the sentence components and position information are not emphasized enough. This article combines Ci-Lin and word2vec to divide all of the words in the question-answer pairs into groups according to the semantics and select one kernel word in each group. The remaining words are common words and realize the semantic mapping mechanism between kernel words and common words. With this Chinese semantic mapping mechanism, the common words in all questions and answers are replaced by the semantic kernel words to realize the normalization of the semantic representation. Meanwhile, based on the bi-directional LSTM model, this article introduces a method of the combination of semantic role labeling and positional context, dividing the sentence into multiple semantic segments according to semantic logic. The weight is given to the neighboring words in the same semantic segment and propose semantic role labeling position attention based on the bi-directional LSTM model (BLSTM-SRLP). The good performance of the BLSTM-SRLP model has been demonstrated in comparative experiments on the food safety field dataset (FS-QA).
\end{abstract}

CCS Concepts: • Theory of computation $\rightarrow$ Automated reasoning;

Additional Key Words and Phrases: Question answering, BLSTM model, semantic positional-based attention, Chinese semantic mapping mechanism

ACM Reference format:

Mingwen Bi, Qingchuan Zhang, Min Zuo, Zelong Xu, and Qingyu Jin. 2021. Bi-directional Long Short-Term Memory Model with Semantic Positional Attention for the Question Answering System. ACM Trans. Asian Low-Resour. Lang. Inf. Process. 20, 5, Article 77 (June 2021), 13 pages.

https://doi.org/10.1145/3439800

This study was supported by the National Key Technology R\&D Program of China (2016YFD0401205), National Natural Science Foundation of China (61702018), Beijing Natural Science Foundation (4202014), and (20YJCZH229).

Authors' addresses: M. Bi, College of Information and Electrical Engineering, China Agricultural University, Key Laboratory of Agricultural Standardization, Ministry of Agriculture and Rural Affairs, Beijing, China; email: 18310960648@ 163.com; Q. Zhang, M. Zuo, Z. Xu, and Q. Jin, National Engineering Laboratory for Agri-product Quality Traceability, Beijing Technology and Business University, Beijing, China; emails: zqc1982@126.com, \{zuomin123, zhiluchuijiao, bolu33571\}@163.com.

Permission to make digital or hard copies of all or part of this work for personal or classroom use is granted without fee provided that copies are not made or distributed for profit or commercial advantage and that copies bear this notice and the full citation on the first page. Copyrights for components of this work owned by others than ACM must be honored. Abstracting with credit is permitted. To copy otherwise, or republish, to post on servers or to redistribute to lists, requires prior specific permission and/or a fee. Request permissions from permissions@acm.org.

(C) 2021 Association for Computing Machinery.

2375-4699/2021/06-ART77 \$15.00

https://doi.org/10.1145/3439800

ACM Trans. Asian Low-Resour. Lang. Inf. Process., Vol. 20, No. 5, Article 77. Publication date: June 2021. 


\section{INTRODUCTION}

As the saying goes: To the people foodstuff is all-important, To the foodstuff safe is all-important. The issue of food safety has been discussed for a long time and has become more prominent in the past decade or so, and has become a topic of widespread concern in the world. However, there is not much official science knowledge in the field of food safety, and most people rely on experience to judge. The Web and books cannot provide users with fast, concise feedback, and uneven answers make people unable to choose. Therefore, the establishment of an intelligent question answering (QA) system in the field of food safety is of great significance. With the rapid development of natural language technology and deep learning, the research on QA has become active and fruitful [1-4]. An intelligent QA system as a convenient way to replace the artificial interaction has been applied to various fields (e.g., Watson, Google Assistant, Microsoft Cortana).

The current technical difficulties of intelligent QA mainly lie in semantic understanding and sentence representation, which is reflected in two aspects, On the one hand, the question and answer cannot exactly match due to the lexical gap (i.e., there may not be any matching kernel words between them, the length of the question-and-answer sentences are not uniform, and the semantics are not uniform). In fact, the lexical gap can easily lead to the target answer being judged as an irrelevant answer. On the other hand, the candidate answers are usually more verbose, accompanied by semantic noise, and have a lower semantic density, with a large amount of semantic ambiguity and text content irrelevant to the question, resulting in some difficulties in generating the correct answer representation.

In view of the problem of lexical gap, researchers propose to utilize QA pairs to learn the translation model [5] and improve the traditional information retrieval model. The basic assumption is that the question-answer pair is "parallel text" and the relationship of words (or phrases) can be established by the translation probability of word to word (or phrase to phrase) [3]. Research shows that statistical translation models receive state-of-the-art performance for QA retrieval.

However, in practice, question and answer pairs are far from "parallel". Furthermore, a large number of experiments show that the model can capture more contextual information after the word-level is upgraded to phrase-level. Although different phrases have different meanings in different contexts, which indicates that the model understand the superficial meaning of similar fuzzy matching words and does not reach the deep understanding of semantic resulting.

In view of the problem of sentence representation, the encoder-decoder mechanism was proposed. The encoder-decoder model successfully solved the problem of machine translation [6, 7]. The nature of encoder-decoder is as follows: the encoder first encodes the query as a hidden representation, then the decoder translates the hidden vectors into a target language. The techniques of machine translation can also be used to map questions and answers in a QA task (i.e., a given question generates an answer that can be considered as the target text of the generated source text). Thus, state-of-the-art sequence-to-sequence models with an encoder-decoder framework have emerged.

Inspired by the success of sequence-to-sequence models with an encoder-decoder framework $[7,8]$, researchers introduced various attention mechanisms to the model to emphasize the kernel semantic content of the sentence. The purpose is to adjust the weights of hidden vectors of sentence representations and promote the QA task to a more precise direction. However, all current attention-based mechanisms ignore the positional context based on the sentence component. In information retrieval [9], the positional context has been extensively studied to improve performance. The presence positions of query words are modeled utilizing various kernels and then combined with the traditional information retrieval model to improve retrieval performance. 
This article will present further discussion on the preceding lexical gap and the issue of sentence representation. There are two main aspects. First, synonyms-subject replacement mechanism based on Ci-Lin ${ }^{1}$ (i.e., a dictionary of Chinese synonyms compiled by experts) was introduced to the current state-of-the-art encoder-decoder model to solve the semantics of words previously ignored by researchers. Second, to optimize the current state-of-the-art attention mechanism, semantic role labeling was introduced to enhance the focus on the classification of sentence components and semantic logic location, and to construct the position-aware attention mechanism marked by semantic roles.

We review the related work from the following two main aspects.

Question-and-answer matching: To solve the lexical gap between the question and answer for question-and-answer matching, some previous work focuses on the syntax matching between questions and answers. In the work of Wang et al. [10], quasi-synchronization syntax is used to match tree-dependent QA pairs. A tree kernel function with a logistic regression model is proposed by Heilman and Smith [11]. Other researchers have focused on bridging the lexical gap between questions and answers by learning various modes of translation. Jeon et al. [12] proposed a word-based approach for problem retrieval and evaluated the probabilities of translation-based retrieval models by calculating the similarity between answers in the document. The experimental results show that the proposed translation-based language model is significantly superior to the traditional method. In the work of Zhou et al. [13], when word level is upgraded to phrase level, the phrase-based translation model can capture more contextual information than the wordbased translation model, and its translation accuracy is also better than the word-based translation model, which can improve performance of the retrieve model.

Generated sentence representation: To weaken the semantic interference caused by irrelevant content in sentences, the combined hidden vectors generate accurate sentence representation. Previous work focuses on introducing various attention mechanisms in the hidden layer [14-17]. Li et al. [18] proposed a context-aware attention model. The essence of this model is embedding the problem into a vector $u$ using the location attention mechanism when inputting a document and a question, then using concatenation-based attention (the previous sentence and the current sentence concatenation) + similarity with $u$ to calculate attention score modeling. As a result, the embedding of each sentence was obtained. In the work of Tan et al. [15], the attention of the hidden vector of the answer depends on the hidden vector representation of the problem. Santos et al. [14] proposed a two-way attention mechanism. The attentive weight of the question (answer) is affected by the answer (question) expressed in terms of the word-by-word interactive matrix. However, these attentions depend on the hidden vectors, which lead to excessive expression of sentences. At the end of the sentence, the word sequence has a lot of semantic accumulation due to the word sequence of RNN (or variant of RNN). To remedy this bias of the attention mechanism, Wang et al. [16] proposed three methods of intrinsic concern that introduced attentive information to the hidden representation and achieved state-of-the-art performance in the QA field.

To the best of our knowledge, all of the mentioned proposed models in the preceding two aspects ignore or fail to emphasize the following two issues. First, the problem of synonym matching is neglected when solving the sentence matching problem caused by the lexical gap. Second, there is ignorance of the positional context when utilizing an attention mechanism for generating sentence representations, which has been extensively studied in terms of performance improvement in information retrieval. In the work of Liu et al. [19] and Zhao et al. [9], the presence positions of query words are modeled utilizing various kernels and then combined with the traditional information retrieval model to improve retrieval performance.

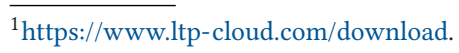


Based on the preceding two issues, we propose a novel idea. In view of the natural language semantic description of diversification and the difficulty of semantic alignment, this article proposes a method of semantic normalization (i.e., reduce the vocabulary gap between synonyms) by combining Chinese features with word2vec and semantic Ci-Lin. Specifically, we divide all words in the question-answer pairs into groups according to the semantics and select one kernel word in each group. The remaining words are common words, and they realize the semantic mapping mechanism between kernel words and common words. With this semantic mapping mechanism, the common words in all questions and answers are replaced by the semantic kernel words to realize the normalization of the semantic representation. In addition, they strengthen the ability of the model to deeply understand sentence semantics. In view of the issue of positional awareness ignored by previous researchers, the BLSTM-SRLP model is proposed. When a question word appears in the answer sentence, it will have an effect on the neighboring words in its sentence component. We will discuss this in detail in the next section. We address neglected positionalaware problems by combining semantic role annotation and position-attention mechanisms. The large number of experiments show that our model is better than the state-of-the-art model.

\section{EXPERIMENTS}

\subsection{Description of BLSTM-SRLP}

In this section, we introduce our approach for QA tasks. Suppose that if a word in the question appears in the answer sentence (we call it a question word), the question word in the answer sentence will have an effect on its neighbors. In other words, the neighbors should be given more attention than those distant words, because they represent more problem semantics. Based on this assumption, we propose the BLSTM-SRLP model to simulate the position-aware influence of each sentence component in the answer sentence by the core word in a question. To be specific, we first utilize natural language processing techniques marked by semantic roles to process answer sentences, divide the sentence into different sentence components, and number them. Then we locate the question word in each sentence component. We propose a position-aware influence propagate strategy, in which the influence of the question word on the neighboring words varies with distance in the same sentence component. In other words, such influence can only be propagated within each sentence component, and when the influence propagates to the boundary of the sentence component, the communication is interrupted. In short, the transmission cannot cross the sentence component boundary. We simulate the sematic effects of the question words in the answer sentence on the neighboring words by accumulating the semantic state that the whole sentence is affected by the position awareness. Finally, we merge the strategy with the traditional attention mechanism and weight the hidden vectors of each word to get the answer representation.

\subsection{Framework of BLSTM-SRLP}

The QA system, we propose can be regarded as an input-question-answer triplet, which denotes that users submit queries through the system, questions and corresponding answers in the corpus consisting of question-answer pairs. Later, Figure 3 shows the framework of our positional attention based on BLSTM (BLSTM-SRLP) for answer representation $r_{a}$. To have a better comparison, we utilize the sematic attention mechanism based on the traditional attention mechanism for submitted query (input) representation $r_{i}$, and the framework of the model is shown in Figure 1 . As the QA system requires a quick response to the user's question, in ensuring the premise of the effect, a relatively fast algorithm should be utilized as much as possible in the online program. Thus, we model the user input sentence using the BLSTM model, embed the trained words as input, and generate the hidden vectors. 


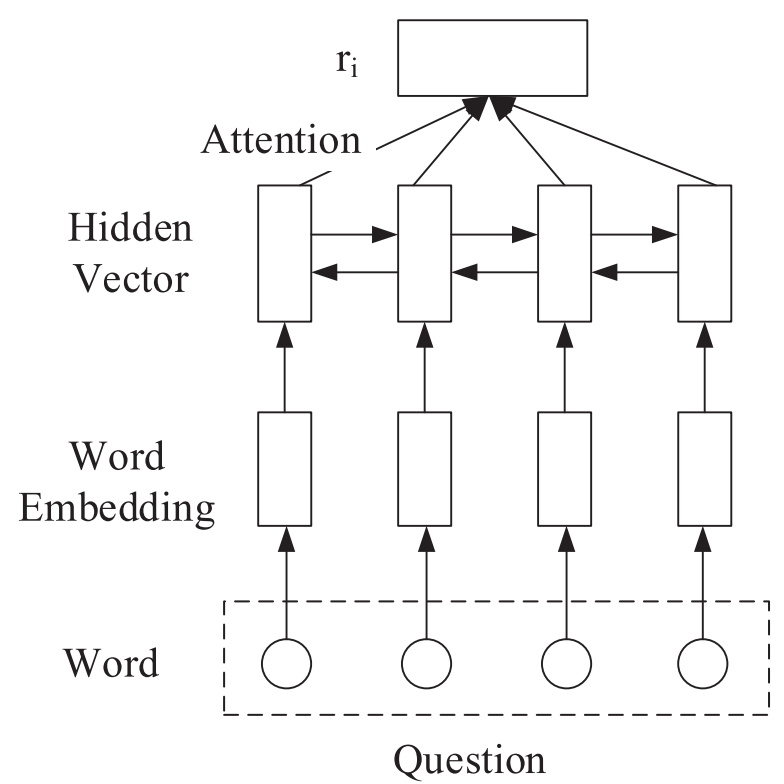

Fig. 1. Input representation with classical attention based on BLSTM.

To get the problem representative, the same traditional attention-based attention mechanism is used, which only relies on the hidden vectors for the weight of attention. Specially, the problem representative is not the final version. The final version of the problem representative will be covered in detail in the next section. An SRL-based approach is proposed for answer representation, which performs additional steps further than the traditional attention mechanism as follows. First, divide the sentence components of the answer sentence by semantic roles. Second, position the question word in each sentence component of the answer. Third, propagate the influence of the question word on neighboring words within the sentence component by our proposed position-aware propagation strategy. Fourth, generate a vector for each word based on positionaware influences by propagating the influence. Fifth, amalgamate position-aware vectors with the traditional attention mechanism for answer representation.

2.2.1 Offline Program (Batch). In this section, we discuss the system's offline programs and the QA's final version of $Q$ in the corpus in detail. In addition, we illustrate the system's question-andanswer matching mechanism.

The main purpose of the QA system we developed in the field of food safety is to quickly and accurately make accurate and concise responses to user-supplied food safety-related inputs. The system-used dataset is independently developed by us in the field of food safety (i.e., FS-QA). We propose a strategy based on I-Q matching. To be specific, the user first inputs the query, then the system matches the input of the user with the QA pairs in the corpus, and then outputs the corresponding answer after the match succeeds. We found that the key point of the proposed I-Q matching strategy is whether $Q$ in the corpus can cover the food safety knowledge that most users usually need, and has nothing to do with $A$. However, we found a situation in which much of the knowledge contained in $A$ exceeds the question semantics contained in question $Q$ in the corpus. Thus, we extract the excess semantics of $A$ as a supplement $A^{\prime}$ to the question of $Q$ (i.e., extended knowledge beyond the question used in the answer sentence). Through such an operation, the answer is fully utilized, meanwhile expanding the coverage of the problem. Inspired by $A$ 's ability 


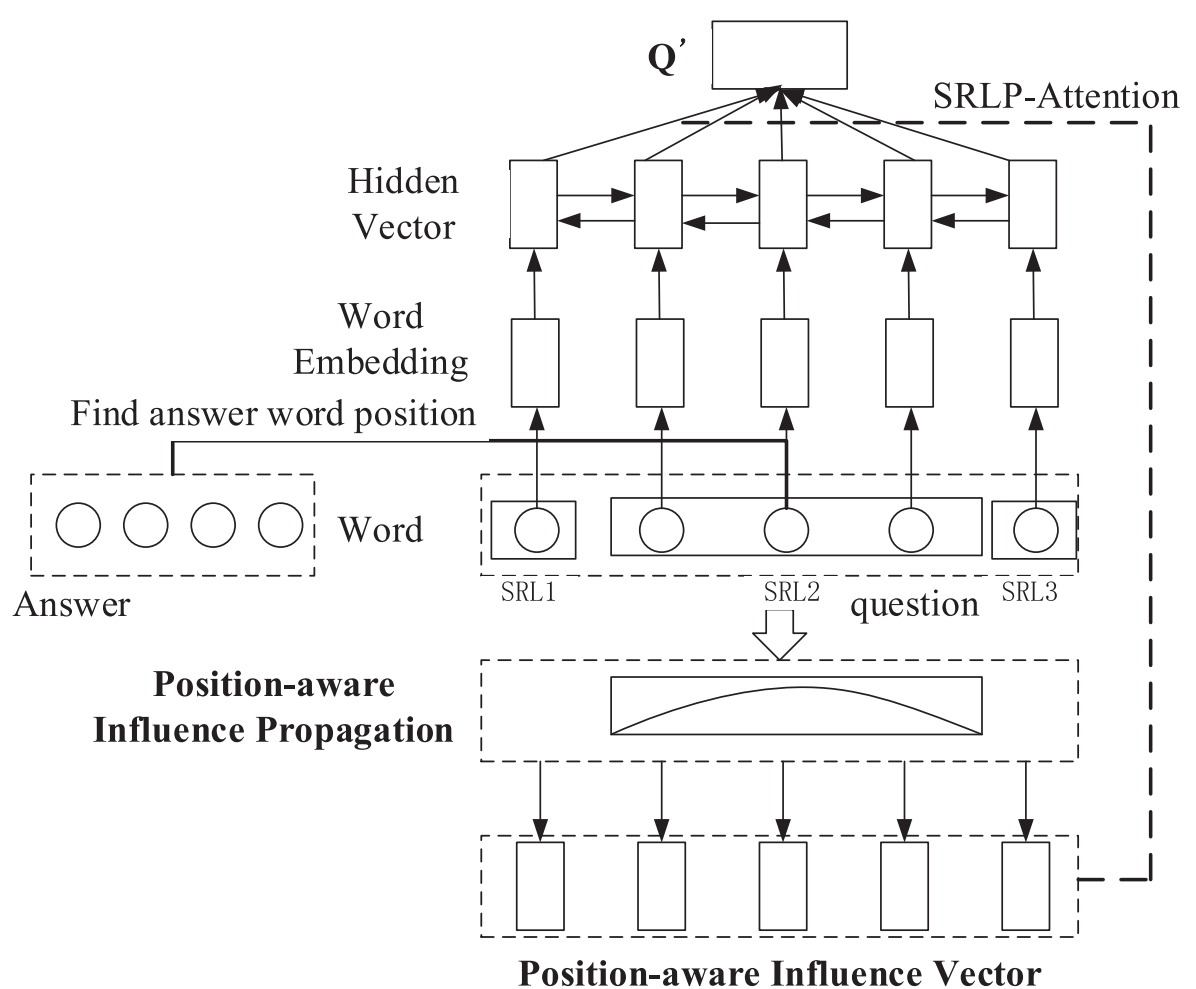

Fig. 2. Question representation with SRLP attention based on BLSTM.

to influence $Q$, combining the question words in $Q$ affects the generation of the answer vector representation mentioned previously, and we utilize the answer word in the answer to influence the generation of a question's sentence representation. The framework is shown in Figure 2. In fact, Figure 2 is the reaction of Figure 3. Through the preceding operation, the question representation $Q^{\prime}$ was obtained. By accumulating the $Q^{\prime}$ and $A^{\prime}$, the final version $r_{q}$ of question representation was completed.

$$
r_{q}=Q^{\prime}+A^{\prime}
$$

Through the attentive representations of both input and the question, there are many similarity functions that can be utilized to measure the correlation between them. We utilize the Manhattan distance similarity function with L1-norm (Formula (2)), which outperforms other alternatives (e.g., cosine similarity).

$$
\operatorname{sim}\left(r_{i}, r_{q}\right)=\exp \left(-\left\|r_{i}-r_{q}\right\|_{1}\right)
$$

2.2.2 Position-aware Influence Propagation. Based on our previous assumptions, if a question word appears in the answer sentence, it will have an effect on its neighboring context. We utilize Gaussian kernel functions to model position-aware influence propagation. Gaussian kernel function has proved to be quite effective in the information retrieval model.

$$
\operatorname{Kernel}(u)=\exp \left(\frac{-u^{2}}{2 \sigma^{2}}\right),
$$




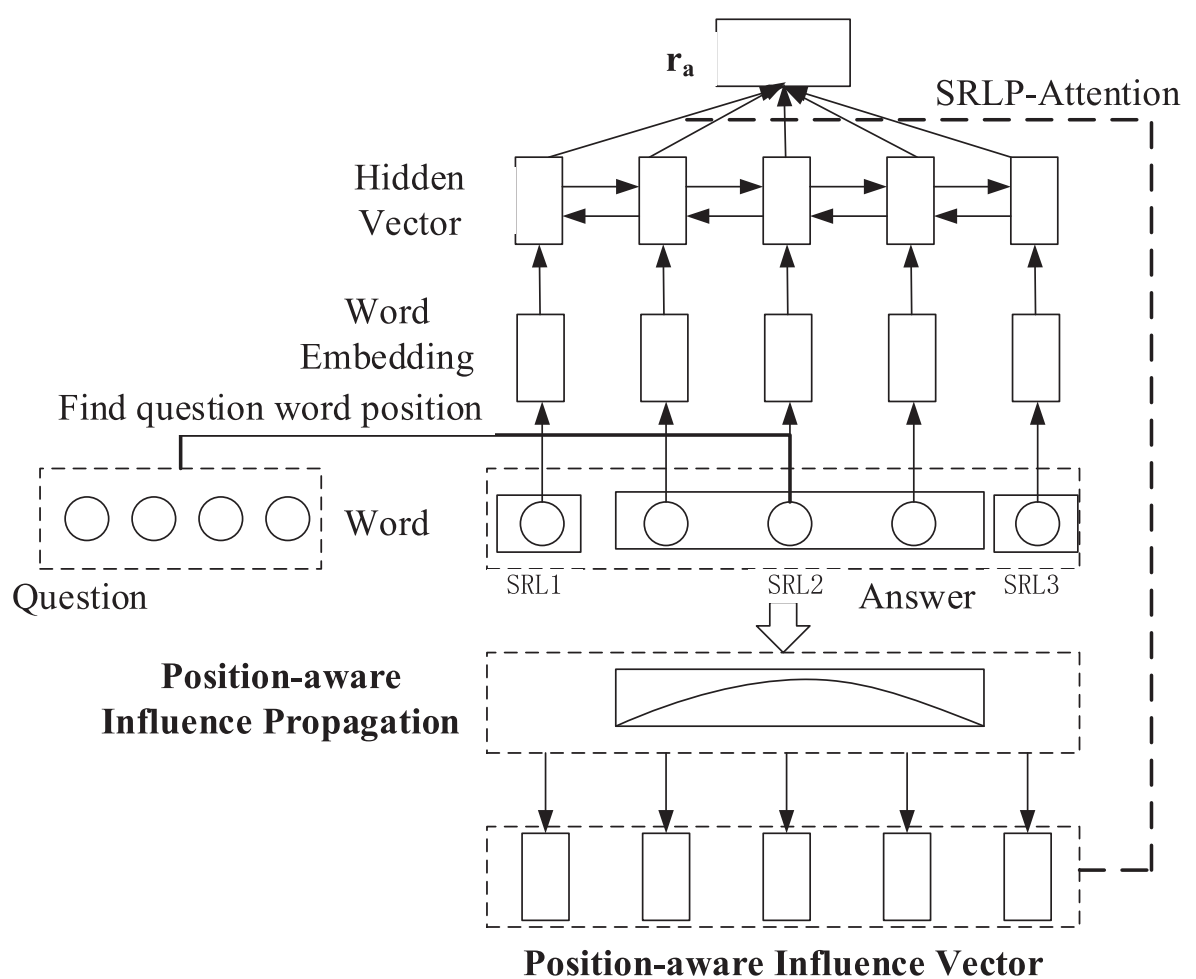

Fig. 3. Answer representation with SRLP attention based on BLSTM.

where $u$ denotes the distance between the question word and the current word, and $\sigma$ is a parameter that constrains the propagation range. In addition, Kernel $(u)$ indicates the corresponding effect based on the kernel distance $u$.

Pay special attention to the position-aware influence diminishing as the distance increases. In particular, when $u=0$ (i.e., the current word is exactly a question word), the propagation of influence reaches its peak. For the propagation range $\sigma$, the optimal value may vary from word to word. In this work, we utilize the same constant value $\sigma$ for all question words and focus on incorporating position awareness into the attention mechanism.

2.2.3 Position-aware Influence Vector. In this section, to model the effects of attention in a highdimensional space, we demonstrate how to obtain the position-aware influence vector of each word in the answer sentence. We first assume that the effect on a particular distance follows the Gaussian distribution in the hidden dimension, which has been proven to be effective and practical in previous research work [20]. Then, based on this assumption, the influence basic matrix $K$ is defined, where each column indicates the influence basic vector corresponding to a specific distance. More formally, each element of $K$ is defined as follows:

$$
K(i, u) \sim N\left(\operatorname{Kernel}(u), \sigma^{\prime}\right)
$$

where $K(i, u)$ denotes the influence of the distance $u$ in the $i$-th dimension, and $N$ is the normal density with the expected value of the $\operatorname{Kernel}(u)$ value and the standard deviation $\sigma^{\prime}$. 
Utilizing the influence matrix, we can obtain the influence vectors of words at a specific position by accumulating the effects of all question words that appear in the answer:

$$
p_{j}=K c_{j}
$$

where $p_{j}$ denotes the cumulative influence vector of the word at position $j$, and $c_{j}$ is a distance counting vector used to measure the number of question words at various distances. Specifically, for a word at position $j$, the count of question words at distance $u\left(\right.$ i.e., $\left.c_{j}(u)\right)$ is calculated as follows:

$$
c_{j}(u)=\sum_{q \in Q}\left\{[(j-u) \in P(q)] e_{j}(j-u)+[(j+u) \in P(q)] e_{j}(j+u)\right\}
$$

where $Q$ represents a question contains a plurality of question words, $q$ is a word in $Q$, and $P(q)$ represents a set of positions where $q$ appears in the answer sentence. In addition, $\left[{ }^{*}\right]$ is an indicator function, equal to 1 if the condition is satisfied or 0 otherwise. $e_{j}(*)$ is a judgment function for whether the current position $j$ and the question word at various distances from $j$ have the same sentence component. Specifically, whether the question word at the distance $u$ and the word at the position $j$ have the same sentence component (i.e., $e_{j}(j \pm u)$ ) is calculated as follows:

$$
e_{j}(j \pm u)=i f(\operatorname{srl}(j), \operatorname{srl}(j \pm u)),
$$

where if $(*)$ is an indicator function, equal to 1 if the two words have the sentence component and otherwise equal to $0 . \operatorname{srl}(*)$ extracts the sentence component number of the word located in the position, and the sentence component number of each word is obtained and stored when the sentence is semantic role labeling.

2.2.4 Positional Attention. In most previous attention mechanisms, the weight of a word's attention depends on the hidden representation; however, position information has not been well studied. In this section, we present a positional attention approach that incorporates the positionaware effects of the question word into the attentiveness of the answer. Specifically, the attention of the word at position $j$ in the answer sentence is formulated as follows:

$$
\alpha_{j}=\frac{\exp \left(a\left(h_{j}, p_{j}\right)\right.}{\sum_{k=1}^{l} \exp \left(a\left(h_{k}, p_{k}\right)\right)},
$$

where $h_{j}$ is the hidden vector based on position $j$ of the BLSTM, $p_{j}$ is the accumulated positionaware influence vector obtained from Equation (5), and $l$ indicates the number of words in the sentence. In addition, $a(*)$ is a scoring function to measure the word importance based on the hidden vectors and the position-aware influence vectors. More formally, the scoring function is defined as follows:

$$
a(i, l)=v^{T} \emptyset\left(W_{H} h_{j}+W_{P} p_{j}+b_{1}\right)+c_{1},
$$

where $W_{H}, W_{P}$ are matrices, $b_{1}$ is a bias vector, they are the parameters of the first layer, $\emptyset(*)=$ $\max (0, x)$ is the $R u L U$ function, which was found better than the monolayer sensor with hyperbolic tangent nonlinearity. $v$ is a global vector, and $v^{T}$ represents its transpose, $c_{1}$ is a bias vector, and they are second-level parameters.

With the weight obtained, the answer sentence is represented by the weighted sum of all hidden vectors:

$$
r_{a}=\sum_{j=1}^{l} \alpha_{j} h_{j}
$$


Table 1. Statistics of Datasets

\begin{tabular}{|l|c|c|c|}
\hline Dataset & $\begin{array}{c}\text { Sample Size } \\
\text { (train/dev/test) }\end{array}$ & $\begin{array}{c}\overline{Q L} \\
\text { (train/dev/test) }\end{array}$ & $\begin{array}{c}\overline{A L} \\
\text { (train/dev/test) }\end{array}$ \\
\hline FS-QA & $4,187 / 374 / 352$ & $8.74 / 9.12 / 8.93$ & $27.82 / 28.25 / 29.33$ \\
\hline
\end{tabular}

Note: $\overline{Q L}$ and $\overline{A L}$ represent the average number of words in the question and answer.

\section{RESULTS AND DISCUSSION}

\subsection{Experimental Setup}

Dataset. The experiment we performed used our independently built dataset FS-QA. FS-QA is a question-and-answer corpus dataset for the field of food safety, all of which are collected from major authoritative websites or books (e.g., Global FoodMate, ${ }^{2}$ CIFST $^{3}$ ). The dataset is divided into three parts (i.e., the training set, development set, and test set), and statistical results are presented in Table 1.

Evaluation metrics. To evaluate the performance of the model, we used mean average precision (MAP) and mean reciprocal rank (MRR), which are the main indicators used in QA. In particular, MAP and MRR can be calculated by the following:

$$
\begin{gathered}
M A P=\frac{1}{n} \sum_{q=1}^{n} \operatorname{avg}(P(q)), \\
M R R=\frac{1}{n} \sum_{q=1}^{n} \frac{1}{\operatorname{rank}(q)},
\end{gathered}
$$

where $\operatorname{avg}(P(q))$ is the average precision score of the query $q$, and $\operatorname{rank}(q)$ is the ranking position of the first correct answer in the candidate answer. MRR can be seen as the first correct answer level, and MAP checks all correct answer levels.

Parameter settings. For word embedding, we use the 100-dimensional GloVe word vector. The parameters in BLSTM are set to be shared between questions and answers, and this approach has been shown to be effective in improving performance [16]. The hidden dimension vector and position-aware impact vector are dimensioned to be 50. The propagation range $\sigma$ for the influence (in Equation (3)) is set to 10. $\sigma^{\prime}$ (in Equation (4)) is empirically set to 0.1 . We use cross-entropy loss as a training target, and we use the ADADELTA [22] algorithm to update parameters. We get the best parameters based on the optimal MAP performance on the development set. The loss function graph is shown in Figure 4.

\subsection{Performance Comparison}

To further evaluate the validity of our proposed model, we compared it with recent QA work. We introduced several strong baselines for comparison. Wang and Nyberg [21] employed a threelayer stacked bi-directional LSTM network and federated model are used that combine the stacked BLSTM outputs with the keyword match baseline (BM25). Second, the RNN model based on internal attention added attention before hidden representations [16]. Third, an extended LSTM framework that incorporates $\mathrm{CNN}$ and establishes an attention matrix after the sentence is represented [15]. The preceding models are implemented in Python and comparative experiments on the FSQA. The experimental results are shown in Table 2.

\footnotetext{
${ }^{2}$ http://www.foodmate.net.

${ }^{3} \mathrm{http}: / /$ www.shipinkepu.com.
} 


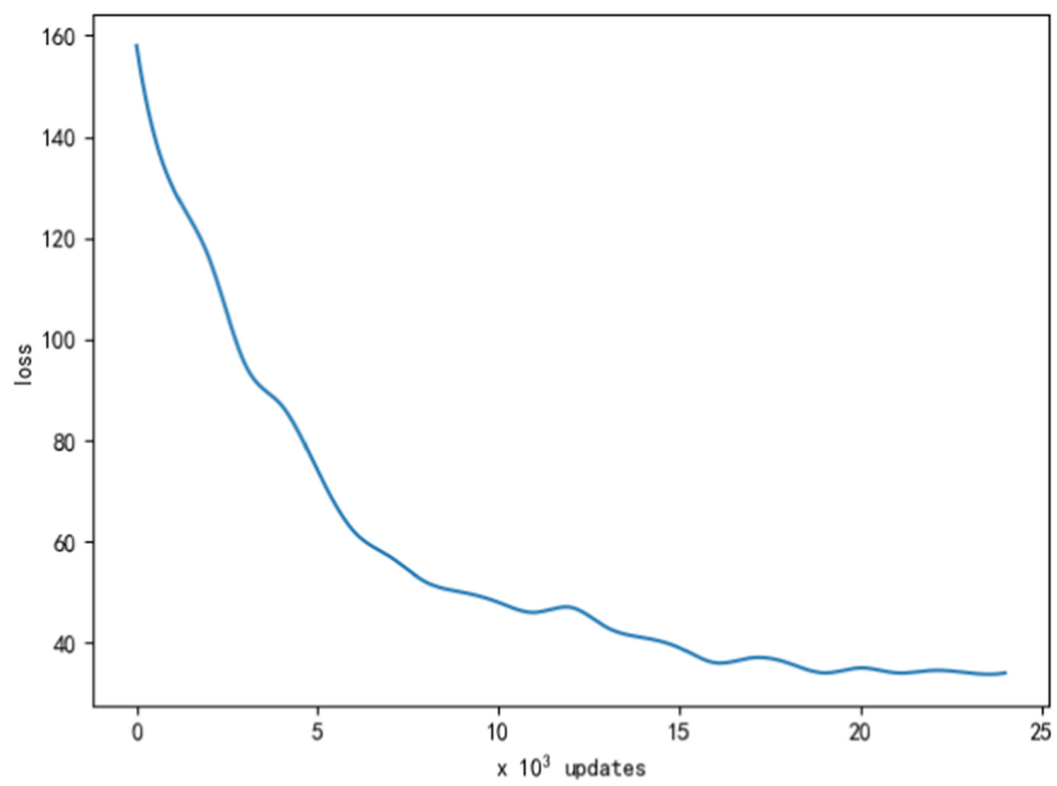

Fig. 4. The loss function graph.

Table 2. Performance Comparison of QA Systems on FS-QA

\begin{tabular}{|l|c|c|}
\hline Model & MAP & MRR \\
\hline BLSTM+BM25 [21] & 0.7364 & 0.8072 \\
Wang et al. (2016) [16] & 0.7544 & 0.8285 \\
Tan et al. (2016) [15] & 0.7720 & 0.8413 \\
LSTM-SRLP & 0.7903 & 0.8662 \\
BLSTM-SRLP & 0.7982 & 0.8701 \\
\hline
\end{tabular}

It is observed that we have achieved state-of-the-art performance on FS-QA. This confirms our previous assumption that the words close to question words in the same sentence component should be given more attention than those far away. In addition, it proves that the answer word has an impact on the generation of question representation.

\subsection{Impact of SRLP Attention}

To investigate the effect of our proposed BLSTM-SRLP attention approach, we introduced two basic baselines that did not use SRLP attention: the approach with weighted average (e.g., the LSTM encoder-decoder model [8] and the RNN encoder-decoder model [7]) and the approach with classical attention (e.g., [21]). The preceding approaches are implemented in Python and comparative experiments on the FS-QA. The experimental results shown in Table 3. Our model performs better than the others.

We observed that the LSTM encoder-decoder model has better performance than the RNN encoder-decoder. LSTM has a greater ability to learn long-range time-dependent data because there is a considerable time lag between these issues and the corresponding key information in their answer sentence. It has also been demonstrated that bi-directional LSTM can match questions 
Table 3. Performance of Various Models on FS-QA

\begin{tabular}{|l|c|c|}
\hline Model & MAP & MRR \\
\hline RNN & 0.6398 & 0.7131 \\
RNN-ATT & 0.7175 & 0.8082 \\
RNN-SFPA & 0.7754 & 0.8477 \\
LSTM-SFPA & 0.7903 & 0.8662 \\
BLSTM-SFPA & 0.7982 & 0.8701 \\
\hline
\end{tabular}

\section{Q: 哪个部门负责食品安全风险评估？}

\section{国务院卫生行政部门通过食品安全风险监测或者接到举报 发现食品可能存在安全隐患的, 应当立即组织进行检验}

(a) Traditional attention

\section{国务院卫生行政部门通过食品安全风险监测或者接到举报 发现食品可能存在安全隐患的, 应当立即组织进行检验}

(b) SRLP

Fig. 5. A specific case of traditional attention and SRLP.

and answers more efficiently because bi-directional LSTM can use previous and future contexts by processing data from both directions. In addition, we noticed that the classical attentional mechanism slightly outperformed the weighted average approach by capturing a portion of the informational lexicon in the answer. However, it cannot give the question words and the neighboring context specific attention in the answers, which can lose a lot of useful information for the QA match. In our proposed SRLP attention mechanism, the importance of the question word and the neighboring context in the answer sentence will have an explicit improvement by the position-aware propagation of the question word. In addition, we were able to achieve significant improvements compared to the baselines on our FS-QA. The MAP maximum improvement rate is as high as $8.07 \%$.

\subsection{A Specific Case}

To explain the BLSTM-SRLP model proposed in this article more intuitively, we utilize a specific QA case to describe the word heat map based on traditional attention and the attention mechanism proposed here, as shown in Figure 5. Specifically, to answer this question, the model will focus more on the word “卫生行政部门 (health administrative department)" in the answer sentence. However, the traditional attention mechanism pays more attention to some irrelevant words: “监测 (monitor),” “安全隐患 (security threat),” and “检验 (inspect).” Although these words often appear in semantic association with “风险 (risk)” and “评估 (estimate),"they do not necessarily play a positive role in QA. In contrast, the model proposed in this article first found the 
question words that appear in the answer sentence. In this case, the words “部门 (department)," “食品安全 (food safety),” and “风险 (risk)” were given more attention, and through the influence propagation, the model can pay more attention to the surrounding context, such as “卫生行政部门,” which provides more valuable clues for QA. Therefore, the SRLP attention presented in this work has better performance than traditional attention.

\section{CONCLUSION}

In this article, we proposed a semantic role labeling positional attention based on the BLSTM (BLSTM-SRLP) model in which the positional context of the question words is included in attentional representations of the answer. In addition, a synonyms-subject replacement mechanism (i.e., that maps common words as kernel words) can effectively bridge the lexical gap. The experimental results on FS-QA demonstrate the superiority of our model over the baselines that do not consider the lexical gap and position information. Furthermore, our SRLP attention mechanism has higher performance than the state-of-the-art approach in QA. We plan to evaluate our model in different tasks and will try to improve it. In the future, the ontology knowledge base will be combined with the model to address ambiguity issues due to a large number of similar symptoms.

\section{References}

[1] A. Abdi, N. Idris, and Z. Ahmad. 2018. QAPD: An ontology-based question answering system in the physics domain. Soft Computing 22, 1 (2018), 213.

[2] A. Figueroa and G. Neumann. 2016. Context-aware semantic classification of search queries for browsing community question-answering archives. Knowledge-Based Systems 96 (2016), 1-13.

[3] G. Zhou, Y. Zhou, T. He, and W. Wu. 2016. Learning semantic representation with neural networks for community question answering retrieval. Knowledge-Based Systems 93 (2016), 75-83.

[4] T. Hao, W. Xie, Q. Wu, H. Weng, and Y. Qu. 2017. Leveraging question target word features through semantic relation expansion for answer type classification. Knowledge-Based Systems 133 (2017), 43-52.

[5] C.-H. Wu, C.-H. Liu, and P.-H. Su. 2015. Sentence extraction with topic modeling for question-answer pair generation. Soft Computing 19, 1 (2015), 39-46.

[6] D. Bahdanau, K. Cho, and Y. Bengio. 2014. Neural machine translation by jointly learning to align and translate. arXiv:1409.0473.

[7] K. Cho, B. V. Merrienboer, C. Gulcehre, D. Bahdanau, F. Bougares, H. Schwenk, and Y. Bengio. 2014. Learning phrase representations using RNN encoder-decoder for statistical machine translation. arXiv:1406.1078.

[8] I. Sutskever, O. Vinyals, and Q. V. Le. 2014. Sequence to sequence learning with neural networks. In Advances in Neural Information Processing Systems, Vol. 3104. Curran Associates, Red Hook, NY, 1-9.

[9] J. Zhao, J. X. Huang, and B. He. 2011. CRTER: Using cross terms to enhance probabilistic information retrieval. In Proceedings of the 34th International ACM SIGIR Conference on Research and Development in Information Retrieval. ACM, New York, NY, 155-164.

[10] M. Wang, N. A. Smith, and T. Mitamura. 2007. What is the Jeopardy model? A quasi-synchronous grammar for QA. In Proceedings of the 2007 foint Conference on Empirical Methods in Natural Language Processing and Computational Natural Language Learning (EMNLP-CoNLL'07). 1-11.

[11] M. Heilman and N. A. Smith. 2010. Tree edit models for recognizing textual entailments, paraphrases, and answers to questions. In Proceedings of the 2010 Annual Conference of the North American Chapter of the Association for Computational Linguistics. 1011-1019.

[12] J. Jeon, W. B. Croft, and J. H. Lee. 2005. Finding similar questions in large question and answer archives. In Proceedings of the 14th ACM International Conference on Information and Knowledge Management. ACM, New York, NY, 84-90.

[13] G. Zhou, L. Cai, J. Zhao, and K. Liu. 2011. Phrase-based translation model for question retrieval in community question answer archives. In Proceedings of the 49th Annual Meeting of the Association for Computational Linguistics: Human Language Technologies. 653-662.

[14] C. D. Santos, M. Tan, B. Xiang, and B. Zhou. Attentive pooling networks. arXiv:1602.03609.

[15] M. Tan, C. Santos, B. Xiang, and B. Zhou. 2015. LSTM-based deep learning models for non-factoid answer selection. arXiv:1511.04108.

[16] B. Wang, K. Liu, and J. Zhao. 2016. Inner attention based recurrent neural networks for answer selection. In Proceedings of the 54th Annual Meeting of the Association for Computational Linguistics (Volume 1: Long Papers). 1288-1297. 
[17] Z. Yang, D. Yang, C. Dyer, X. He, A. Smola, and E. Hovy. 2016. Hierarchical attention networks for document classification. In Proceedings of the 2016 Conference of the Association for Computational Linguistics: Human Language Technologies. 1480-1489.

[18] H. Li, M. Min, Y. Ge, and A. Kadav. 2017. A context-aware attention network for interactive question answering. arXiv:1612.07411.

[19] B. Liu, X. An, and J. X. Huang. 2015. Using term location information to enhance probabilistic information retrieval. In Proceedings of the 38th International ACM SIGIR Conference on Research and Development in Information Retrieval. 883-886.

[20] Y. Zhang, Y. Wang, G. Zhou, J. Jin, B. Wang, and X. Wang. 2018. Multi-kernel extreme learning machine for EEG classification in brain-computer interfaces. Expert Systems with Applications 96 (2018), 302-310

[21] D. Wang and E. Nyberg. 2015. A long short-term memory model for answer sentence selection in question answering. In Proceedings of the 53rd Annual Meeting of the Association for Computational Linguistics and the 7th International foint Conference on Natural Language Processing (Volume 2: Short Papers). 707-712.

[22] M. D. Zeiler. 2012. ADADELTA: An adaptive learning rate method. arXiv:1212.5701.

Received August 2020; revised November 2020; accepted November 2020 